\title{
Cardiovascular Oscillations: In Search of a Nonlinear Parametric Model
}

\author{
Andriy Bandrivskyy ${ }^{a}$, Dmitry Luchinsky $^{a}$, Peter McClintock $^{a}$, \\ Vadim Smelyanskiy ${ }^{b}$, Aneta Stefanovska ${ }^{c a}$, and Dogan Timucin ${ }^{b}$, \\ ${ }^{a}$ Department of Physics, Lancaster University, Lancaster LA14YB, UK \\ ${ }^{b}$ NASA Ames Research Center, MS 269-2, Moffett Field, CA 94035-1000, USA \\ ${ }^{c}$ Group of Nonlinear Dynamics and Synergetics, Faculty of Electrical Engineering, \\ University of Ljubljana, Tržaška 25, 1000 Ljubljana, Slovenia
}

\begin{abstract}
We suggest a fresh approach to the modelling of the human cardiovascular system. Taking advantage of a new Bayesian inference technique, able to deal with stochastic nonlinear systems, we show that one can estimate parameters for models of the cardiovascular system directly from measured time series. We present preliminary results of inference of parameters of a model of coupled oscillators from measured cardiovascular data addressing cardiorespiratory interaction. We argue that the inference technique offers a very promising tool for the modelling, able to contribute significantly towards the solution of a long standing challenge - development of new diagnostic techniques based on noninvasive measurements.
\end{abstract}

Keywords: cardiovascular system, coupled oscillators, inference, stochastic nonlinear models

\section{INTRODUCTION}

It has long been recognized that, even for healthy subjects in repose, the human heart does not beat at a constant rate. Rather, its frequency varies in time. ${ }^{1-3}$ This heart rate variability influences blood flow, blood pressure, and other parameters of cardiovascular regulation, which also vary in time. Moreover, the influence is mutual, because of the feedback mechanisms in the system. As a result, the blood that circulates through the vessels and its pressure are regulated in a highly complex, dynamical way.

A number of noninvasive measurements (e.g. ECG, blood pressure, cutaneous blood flow, respiration) can in principle be used to characterize the state of the human cardiovascular system. However, the extreme complexity of the system, combined with strong intrinsic noise, make it a challenging problem to find a parametric model capable of describing the numerous possible states of the system. The motivation in this search for a model is to gain further insight into the complex control mechanisms responsible for the function of the cardiovascular system and to improve the diagnosis of cardiovascular diseases, especially in their early stages.

A particular effort has been made to understand the role and origin of the oscillations observed in the cardiovascular signals (see reviews ${ }^{1,4}$ ). It is well recognized that there are three distinct oscillatory modes (around 1, 0.3, and $0.1 \mathrm{~Hz}$ for human) and it was recently suggested that two additional noisy oscillatory modes contribute to the dynamics of blood distribution ${ }^{3,5}$ (around 0.04 and $0.01 \mathrm{~Hz}$ ). With different degrees of confidence, each of the oscillations can be related to a particular physiological mechanism and the hope has arisen that they can provide useful measures of the control mechanisms involved in the system. ${ }^{6}$

There are different, sometimes conflicting, ideas of how to explain and model cardiovascular oscillations. An important class of models is based on differential-delay equations which aim to describe feedback control loops present in the system. It was suggested that the baroreflex control loop can be responsible for oscillations around $0.1 \mathrm{~Hz}$ in the arterial blood pressure and the heart rate variability. ${ }^{4,7,8}$ Also e.g. respiratory feedback control mechanism can result in so-called periodic or Cheyne-Stokes breathing. ${ }^{9,10}$

Correspondence to: A. Bandrivskyy (band@lancaster.ac.uk); and/or A. Stefanovska (aneta@osc.fe.uni-lj.si). 


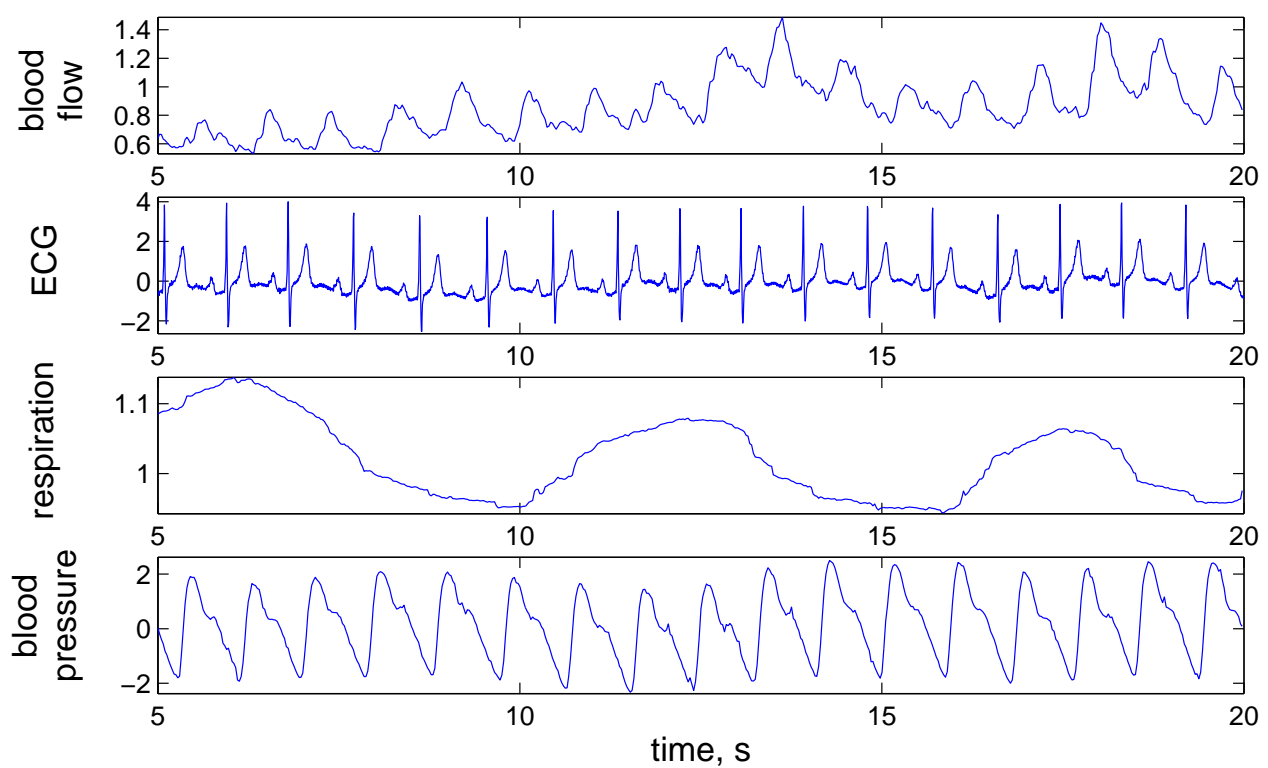

Figure 1. An example of a set of noninvasively measured cardiovascular signals

Another approach is based on the hypothesis that the cardiovascular system can be modelled as a system of coupled oscillators, ${ }^{3,5,11}$ which is consistent with the idea that rhythmical dynamics and oscillators are ubiquitous in nature. ${ }^{12,13}$ However the choice of the model's parameters, e.g. the type of oscillators and the form and strength of the couplings, and the physiological significance to be attributed to them, still remain open problems. Nonetheless, there is a general agreement that the underlying models of the cardiovascular oscillations are necessarily stochastic and nonlinear. ${ }^{6}$ Conventional linear analysis of the oscillations, e.g. wavelet or Fourier transforms, which provide amplitude and frequency information, have been widely used to determine the physiological origin of some of the oscillations, and the changes attributable to cardiovascular diseases. However, for the description of the system's dynamics the essentially nonlinear nature of oscillations, the time-variability of the characteristic frequencies of the oscillations, and the strong intrinsic noise, should also be taken into account. A different approach is required.

In this paper we suggest that fast Bayesian inference of stochastic nonlinear systems ${ }^{14}$ can be applied to the cardiovascular data to infer parameters of the existing models. We present preliminary results, inferring the parameters of a coupled oscillator model from measured cardiovascular data. In particular, we address the well-known cardiorespiratory interaction. ${ }^{15}$ We propose that the inference technique can be further developed to provide a quantitative measure of the success of different models and to illuminate the critical and intricate problem of model choice. We argue that this new approach can be applied to the long standing problem of parameterizing the state of the cardiovascular system, and that it can help to provide deeper insight into the physiological and clinical significance of the model parameters.

In section 2 we describe briefly some cardiovascular signals that can be measured noninvasively. In section 3 we outline the fast Bayesian inference algorithm and show an example of inference for a model system. In section 4 we infer parameters for coupled noise-driven oscillators. In section 5 we show an example of inference applied to the respiratory oscillator using measured data. In section 6 we discuss the possibility of applying the technique to estimate the parameters of cardiorespiratory interaction from the blood flow and respiration signals. Finally, in the conclusion, we list remaining problems and open questions and suggest directions for further research.

\section{NONINVASIVE MEASUREMENTS}

There are well established techniques ${ }^{3}$ for the simultaneous measurement and recording of several kinds of cardiovascular signal from different sites of a body. Noninvasive measurements of ECG, respiration, blood pressure in a finger, cutaneous blood flow and temperature signals were performed. An example of a set of 
measured cardiovascular signals is shown in Fig. 1. The so-called heart rate variability signal, giving a measure of the instantaneous heart rate, can readily be constructed from ECG signal. A big advantage of simultaneous measurements is that very diverse information about the cardiovascular system is being acquired. There is no doubt that the interplay between the different cardiovascular mechanism must be manifested in the cardiovascular signals, and the challenge is to extract this information.

\section{INFERENCE ALGORITHM}

Much nonlinear time series analysis is in practice concerned with the inference of unmeasured or unmeasurable quantities, such as system parameters, from noisy measured time series. The most widely used approach to the problem involves Bayesian inference. However, no general methods are currently available to infer the parameters of a nonlinear model with internal stochastic dynamics. Existing applications of the Bayesian approach rely on heavy numerical methods (like Markov chain Monte Carlo technique) to generate the Bayesian conditional probabilities. ${ }^{16}$ It was recently suggested by Vadim Smelyanskiy that the conditional probability can be obtained by expressing it in terms of a white noise path integral. ${ }^{14}$ Taking advantage of this idea, a concise and fast iterative Bayesian inference scheme can be derived. This technique can be successfully used to infer the parameters of stochastic nonlinear models subject to additive white Gaussian noise.

Consider for the sake of simplicity a one-dimensional dynamical system subject to additive noise of the form

$$
\begin{aligned}
& \dot{x}=K(x \mid c)+\xi(t), \\
& \langle\xi(t)\rangle=0, \quad\langle\xi(t) \xi(0)\rangle=D \delta(t),
\end{aligned}
$$

where $K(x \mid c)$ is the deterministic drift force, $\xi(t)$ is white zero-mean Gaussian noise and $D$ is the noise intensity. We assume that the unknown deterministic force can be represented in the form

$$
K(x \mid c)=\sum_{l=1}^{L} c_{l} f_{l}(x)
$$

where $f_{l}(x)$ are known base functions, and $c=\left\{c_{l}\right\}$ is a set of unknown coefficients that have to be inferred from the experimental data.

We assume that measurement error is negligible compared to the intrinsic dynamical noise $\xi(t)$, and we treat the state variable $x$ as directly observable. Then, using results of, ${ }^{17-19}$ the conditional probability density function $(\mathrm{PDF}) L(x \mid c)$ of an observation $x(t)$ for a given choice of the model parameters $c$ can be written as

$$
L(x \mid c)=P_{\mathrm{st}}\left(x\left(t_{0}\right) \mid c\right) \mathcal{F}_{c}[x(t)] .
$$

Here $P_{\text {st }}(x \mid c)$ is a stationary PDF of the system $(1)$ and $\mathcal{F}_{c}[x(t)]$ is a probability density functional of $x(t)$. It can be expressed through the white noise path integral using the direct interrelation between the noise variable $\xi(t)$ and $x(t)$ given by (1). Then for the probability density functional defined on a time lattice $t_{i}=t_{\text {in }}+i h$ $(i=0,1, \ldots, N)$ with the step $h=\left(t_{\mathrm{fn}}-t_{\mathrm{in}}\right) / N$

$$
\mathcal{F}_{c}[x(t)]=(2 \pi D h)^{-N / 2} \exp \left(-\frac{h}{2} \sum_{i=1}^{N} \frac{\partial K\left(x_{i} \mid c\right)}{\partial x}\right) \exp \left(-\frac{1}{2 D h} \sum_{i=0}^{N-1}\left[x_{i+1}-x_{i}-h K\left(x_{i} \mid c\right)\right]^{2}\right) .
$$

In this derivation we assume that the time step $h$ is sufficiently small $\left(h \ll\left|K_{x}(x \mid c)\right|^{-1}\right)$.

Prior information about the model parameters is contained in a prior $\operatorname{PDF} p(c)$. Given a set of observations one can improve the estimate of the model parameters by computing a posterior PDF $p(c \mid x)$ taking advantage of Bayes' theorem

$$
p(c \mid x)=\frac{L(x \mid c) p(c)}{\int L(x \mid c) p(c) d c}
$$

The integral in the denominator in (5) ensures that the posterior PDF is normalized. Clearly, equation (5) can be applied iteratively each time a new record of measurements is used: the prior distribution in the next iteration 
is just the posterior distribution from the previous iteration. For a sufficiently large number $N$ of observations in $x$ the posterior distribution $p(c \mid x)$ becomes sharply peaked about certain parameter values $c$ corresponding to the most probable model of the system for a given measurement set.

We choose the first prior PDF $p(c)$ as a Gaussian form with respect to the unknown parameters $c$

$$
p(c)=\sqrt{\frac{\operatorname{det} A_{0}}{(2 \pi)^{L}}} \exp \left[-\frac{1}{2}\left(c-c_{0}\right)^{T} A_{0}\left(c-c_{0}\right)\right],
$$

where $c_{0}$ is a vector with arbitrary initial parameter values and $A_{0}$ is a diagonal matrix with arbitrarily small values of diagonal elements reflecting, the fact that the initial values of the model parameters are not known.

$P_{\text {st }}\left(x\left(t_{0}\right) \mid c\right)$ in (3) can be neglected if $N$ is large. Then the likelihood is in the form

$$
L(x \mid c)=(2 \pi D h)^{-N / 2} \exp \left[-\frac{R}{D}-\left(\gamma-\frac{\alpha}{D}\right)^{T} c-c^{T} \frac{B}{2 D} c\right]
$$

where

$$
\begin{aligned}
& \alpha_{k}=\sum_{i}\left(x_{i+1}-x_{i}\right) f_{k}\left(x_{i}\right), \quad x_{i}=x\left(t_{i}\right), \\
& B_{k k^{\prime}}=h \sum_{i} f_{k}\left(x_{i}\right) f_{k^{\prime}}\left(x_{i}\right), \\
& \gamma_{k}=\frac{h}{2} \sum_{i} \frac{\partial f_{k}\left(x_{i}\right)}{\partial x}, \quad R=\frac{1}{2 h} \sum_{i}\left(x_{i+1}-x_{i}\right)^{2} .
\end{aligned}
$$

Representing the exponent in the posterior $\operatorname{PDF} p(c \mid x)$ as a quadratic form with respect to the vector $c$, and minimizing it, one obtains an estimate of the parameters $c$. The width of the distribution $p(c \mid x)$ is the precision of this estimate. The noise intensity can be obtained by minimizing the likelihood (7) with respect to $D$. The algorithm can be applied iteratively: the inferred value of noise intensity $D$, parameters $c$ and the width of their distribution are updated with each new block of data.

The formulation of the algorithm can easily be extended to the $M$-dimensional case with a general noise mixing matrix.

\section{INFERENCE OF PARAMETERS OF COUPLED NOISE DRIVEN OSCILLATORS}

\subsection{Inference in normal coordinates}

The system of $M$ noise driven coupled two-dimensional oscillators can be written in an almost general form as

$$
\begin{aligned}
& \dot{x}_{m}=F_{m}^{x}\left(x_{m}, y_{m}\right)+G_{m}^{x}\left(x_{m}, y_{m}, x_{k}, y_{k}\right)+\xi_{m}^{x}(t), \\
& \dot{y}_{m}=F_{m}^{y}\left(x_{m}, y_{m}\right)+G_{m}^{y}\left(x_{m}, y_{m}, x_{k}, y_{k}\right)+\xi_{m}^{y}(t),
\end{aligned}
$$

where $m, k=\overline{1, M}, k \neq m$. Functions $F_{m}^{x}, F_{m}^{y}$ define the intrinsic properties of each oscillator and $G_{m}^{x}, G_{m}^{y}$ represent couplings between them. $\xi_{m}^{x}(t), \xi_{m}^{y}(t)$ are sources of white Gaussian noise. If the base functions of $F_{m}^{x}, F_{m}^{y}, G_{m}^{x}, G_{m}^{y}$ are known, and if all the dynamical variables $x_{m}, y_{m}$ are measured, one can infer all of the unknown parameters of the system (including noise intensities).

To illustrate the technique we show the result of inference of a model of two coupled noise driven Van der Pol oscillators

$$
\begin{aligned}
& \dot{x}_{m}=y_{m}, \\
& \dot{y}_{m}=-\omega_{m}^{2} x_{m}+\eta_{m}\left(1-x_{m}^{2}\right) y_{m}+g_{m} x_{k}+\xi_{m}(t),
\end{aligned}
$$


where $m, k=\overline{1,2}, m \neq k$. In this case the general inference algorithm can effectively be reduced to one dimensional form. The model for inference can be defined separately for each oscillator

$$
K_{m}\left(x_{m}, y_{m}, x_{k}\right)=\sum_{l=1}^{3} c_{l} f_{l}\left(x_{m}, y_{m}, x_{k}\right)
$$

where $f=\left\{x_{m},\left(1-x_{m}^{2}\right) y_{m}, x_{k}\right\}$. We infer parameters $c_{l}$ and noise intensity $D$ from numerically generated time series of the model (10)-(11). Phase portraits of the two oscillators and the result of inference of the parameters of one of them $(m=1)$ are shown in Fig. 2 and Fig. 3. One can see that all the parameters (including coupling coefficient and noise intensity) converge to their correct values.
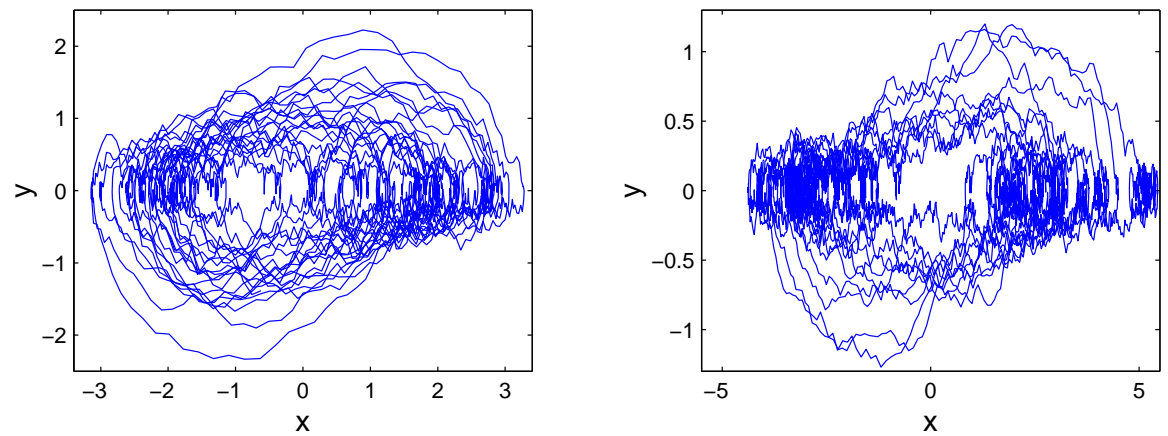

Figure 2. Phase portraits of the model oscillators. Parameters are: $\omega_{1}^{2}=0.25, \eta_{1}=0.2, g_{1}=0.1, D_{1}=0.1$ (left); $\omega_{2}^{2}=0.01, \eta_{2}=0.04, g_{2}=0, D_{2}=0.02$ (right).

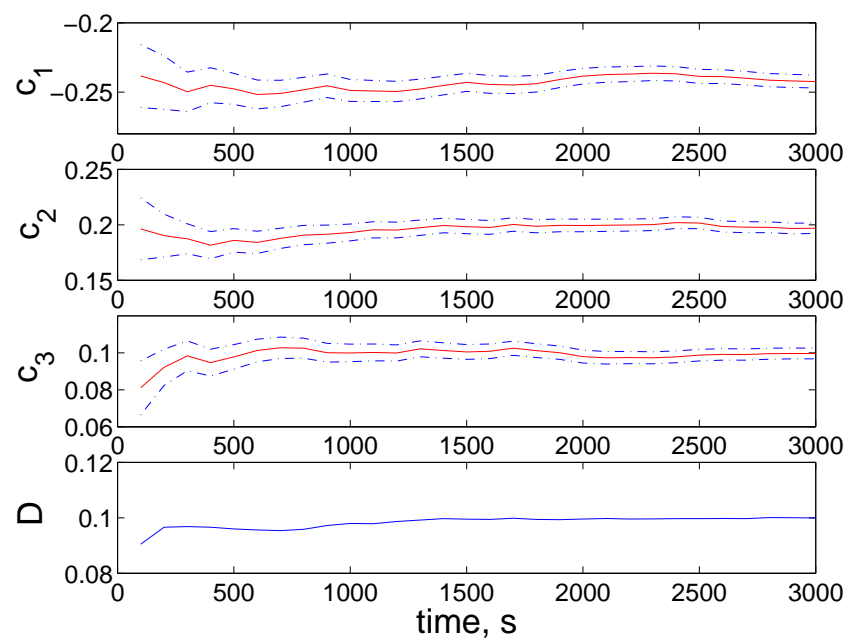

Figure 3. Inference of parameters of one of the oscillators $(m=1)$ in the model (10)-(11). By dashed lines we show width of the parameters distribution.

\subsection{Inference in amplitude and phase coordinates}

Poincaré-like oscillator has been proposed as a basic unit in the model of the cardiovascular system. ${ }^{11} \mathrm{~A}$ coupled oscillator model can be also formulated in terms of amplitude and phase coordinates. Then the couplings and noise are additive terms in the equations for amplitude and phase.

The transformation from $(x, y)$ to $(A, \phi)$ variables is defined as

$$
A_{m}=\sqrt{x_{m}^{2}+y_{m}^{2}}, \quad \phi_{m}=\arctan \left(-y_{m} / x_{m}\right) .
$$


As an example we show inference results for a pair of coupled Poincaré oscillators

$$
\begin{aligned}
& \dot{\phi}_{m}=\omega_{m}+a_{m} \sin \phi_{k}+\xi_{m}^{\phi}(t), \\
& \dot{A}_{m}=\eta_{m}\left(A_{m}-A_{m}^{3}\right)+b_{m} \sin \phi_{k}+\xi_{m}^{A}(t),
\end{aligned}
$$

where $m, k=\overline{1,2}, m \neq k$. The model for inference again can be formulated separately for each oscillator

$$
K_{m}^{\phi}\left(\phi_{m}, A_{m}, \phi_{k}\right)=\sum_{l=1}^{3} c_{l}^{\phi} f_{l}^{\phi}\left(\phi_{m}, A_{m}, \phi_{k}\right), \quad K_{m}^{A}\left(\phi_{m}, A_{m}, \phi_{k}\right)=\sum_{l=1}^{3} c_{l}^{A} f_{l}^{A}\left(\phi_{m}, A_{m}, \phi_{k}\right),
$$

where $f_{m}^{\phi}=\left\{1, \quad \sin \phi_{k}, \quad \cos \phi_{k}\right\}, f_{m}^{A}=\left\{A_{m}, A_{m}^{3}, \quad \sin \phi_{k}\right\}$. We infer parameters $c_{l}^{\phi}, c_{l}^{A}$ and noise intensities $D^{\phi}, D^{A}$ from numerically generated time series of the model (12)-(13). Phase portraits of two oscillators and the result of inference of the parameters of one of them $(m=1)$ are shown in Fig 4 and Fig. 5. Again all the parameters converge to their correct values.
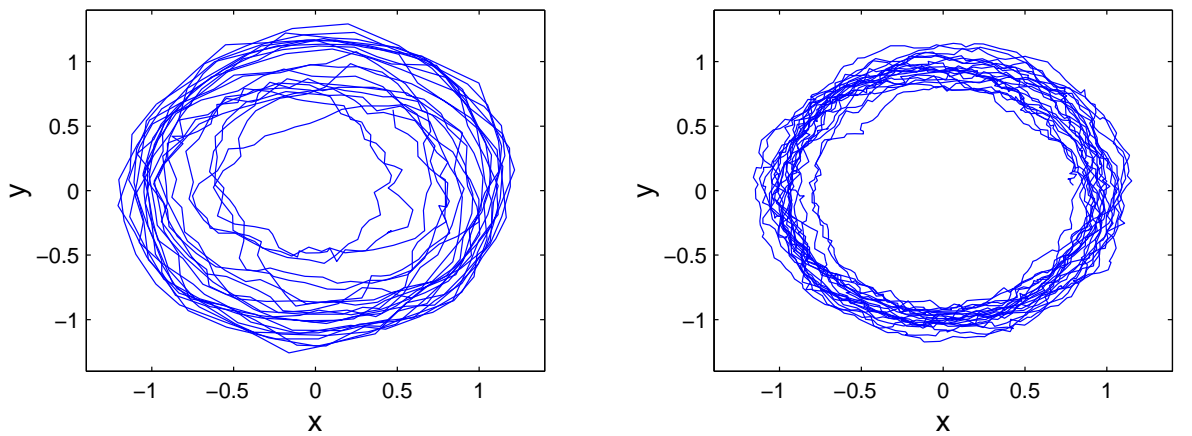

Figure 4. Phase portraits of the model oscillators. Parameters are: $\omega_{1}=7, \eta_{1}=5, a_{1}=1.5, b_{1}=2, D_{1}^{\phi}=0.1, D_{1}^{A}=0.1$ (left); $\omega_{2}=2, \eta_{2}=0.5, a_{2}=0, b_{2}=0, D_{2}^{\phi}=0.02, D_{2}^{A}=0.02$ (right).

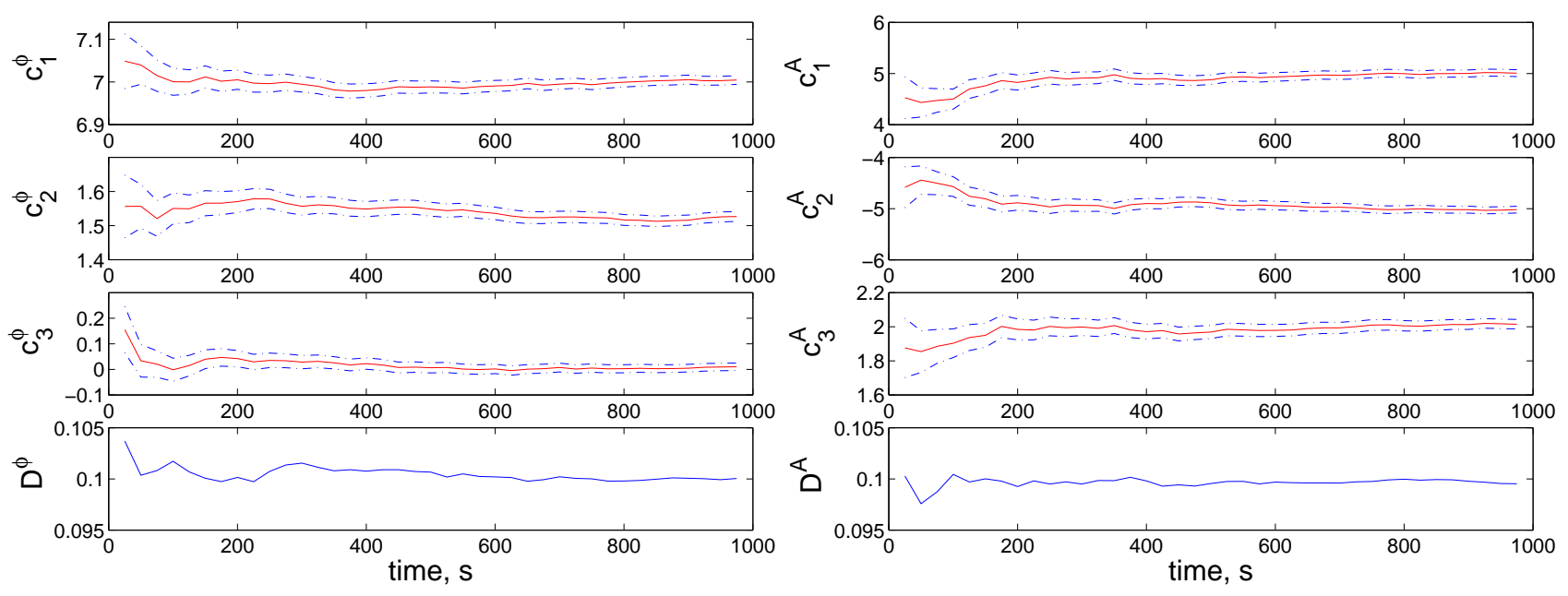

Figure 5. Inference of parameters of one of the oscillators $(m=1)$ in the model (12)-(13).

\section{INFERENCE OF THE RESPIRATORY OSCILLATOR}

To test the ability of the inference technique to deal with real cardiovascular data we apply it to what is apparently the simplest oscillator of the cardiovascular system - the respiratory oscillator. 
The most interesting physiological information is hidden in the variations of the amplitude and phase. So it seems natural to consider a Poincaré-like oscillator, where the equations for amplitude and phase are uncoupled

$$
\dot{\phi}=w, \quad \dot{A}=\eta\left(A-A^{3}\right) .
$$

The normal state variables are given by

$$
x=A \cos \phi, \quad y=-A \sin \phi
$$

From the normalized measured respiration signal $x$ shown in Fig. 8 (top, left) we need to construct both amplitude and phase signals. This can be done approximately* with help of the Hilbert transformation. The constructed $A$ and $\phi$ signals are shown in Fig. 6
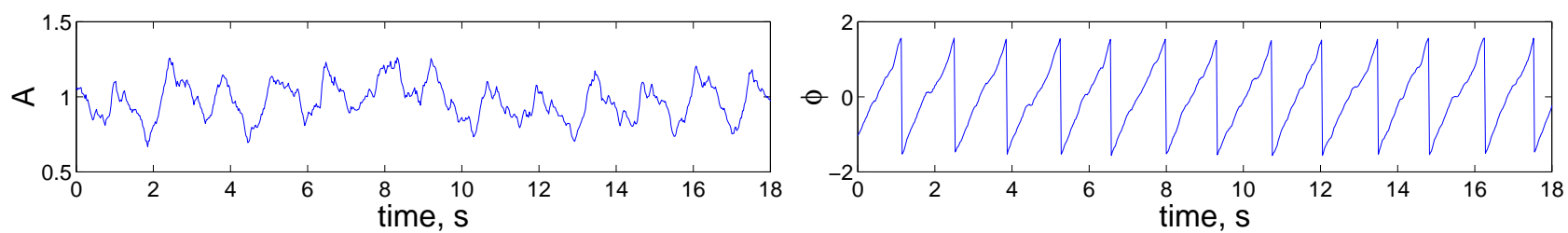

Figure 6. Amplitude and phase of the respiration signal

Apart from the "Poincaré-like" behavior, the fluctuations of $A$ and $\phi$ both have obvious additional periodic components. They result from the non-sinusoidal shape of the respiratory oscillation, which we try to model as being caused by the phase of the oscillator. Then the model for inference is as follows:

$$
\begin{aligned}
& \dot{\phi}=c_{1}^{\phi}+c_{2}^{\phi} \cos (2 \phi)+c_{3}^{\phi} \sin (2 \phi)+\xi^{\phi}(t), \\
& \dot{A}=c_{1}^{A} A+c_{2}^{A} A^{3}+c_{3}^{A} \cos (2 \phi)+c_{4}^{A} \sin (2 \phi)+\xi^{A}(t),
\end{aligned}
$$

where $\xi^{\phi}(t)$ and $\xi^{A}(t)$ are two independent sources of white Gaussian noise of intensities $D^{\phi}$ and $D^{A}$.

The results of inference for all of the parameters are shown in Fig. 7.

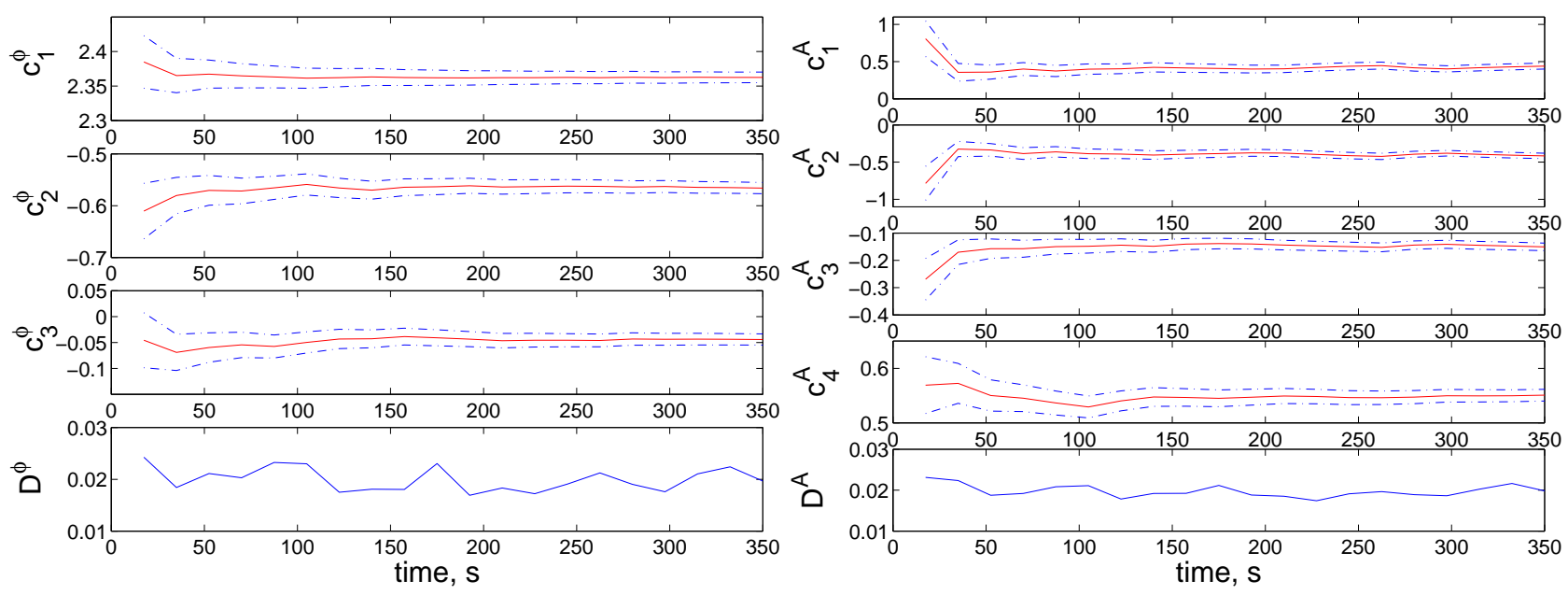

Figure 7. Inference of the respiratory oscillator due to model (14)-(15) using measured respiration signal

To check the results obtained, we generate time-series of the model (14)-(15) numerically, using the inferred parameters. The original respiration signal, the model signal, their power spectral density (PSD) and phase portraits are shown in Fig. 8.

\footnotetext{
*In general it is impossible to reconstruct variables $A$ and $\phi$ for a noisy signal exactly from only one measured variable $x$. The second dynamical variable $y$ can be obtained only approximately with help of the Hilbert transform.
} 

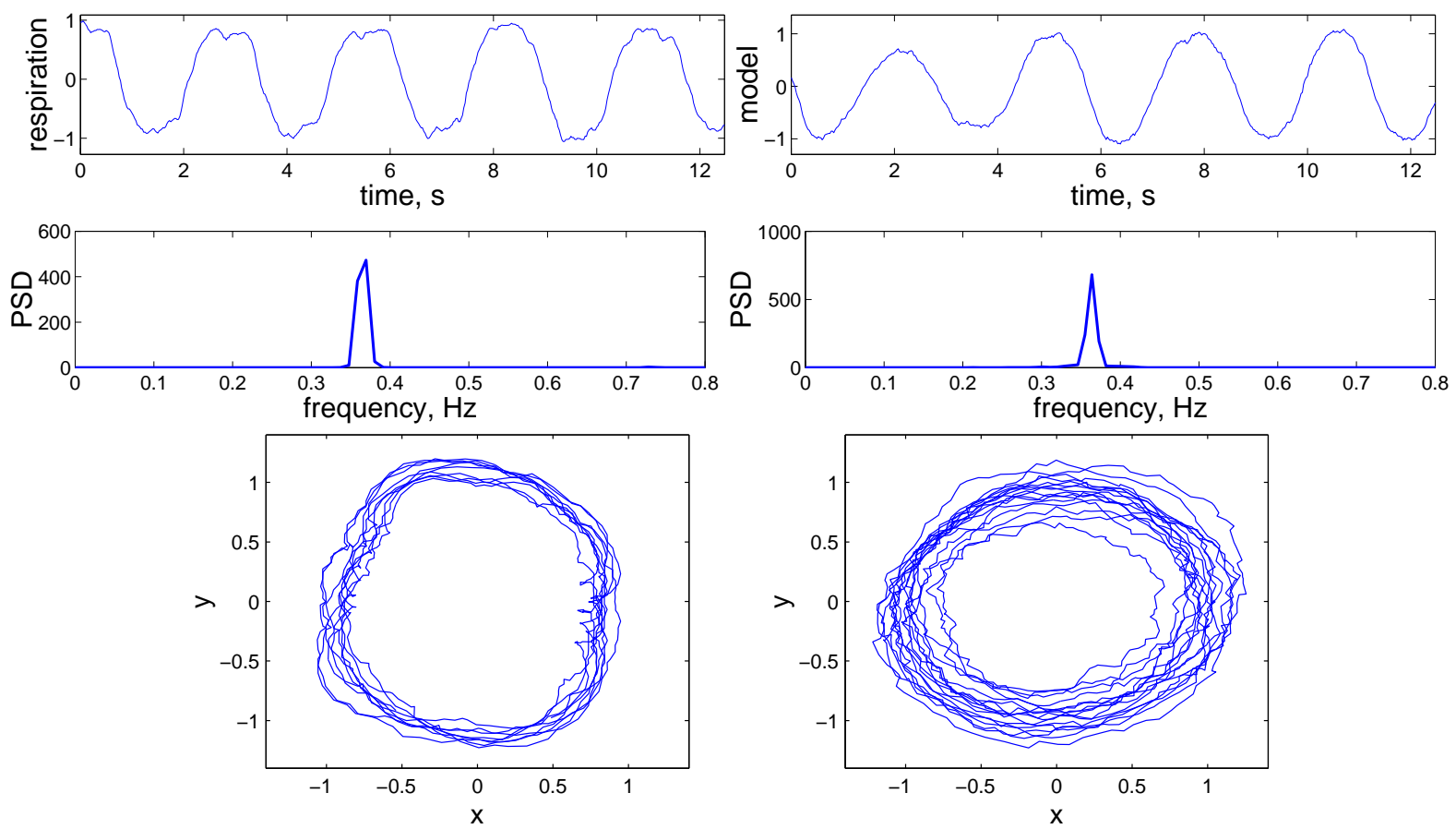

Figure 8. The original respiration signal (left), the model signal (right), their power spectral density (PSD) and phase portraits

\section{INFERENCE OF THE CARDIORESPIRATORY INTERACTION}

In this section we explore the possibility of inferring parameters of the cardiorespiratory interaction from the measured cardiovascular data.

The existence of the cardiorespiratory interaction, i.e. the interaction between the lungs and the heart, has been well known for many years (see review ${ }^{15}$ ). Two important results of this interaction are respiratory-sinus arrhythmia, which means modulation of the heart rate by the respiration; and cardiorespiratory synchronization, meaning that the heart and respiration can synchronize for brief episodes of time. Understanding this interaction is very important in understanding the basic mechanisms of the cardiovascular system. A method for estimation of the strength of the coupling between heart and lungs from measured time series was was recently introduced. ${ }^{20}$

To infer the parameters of the cardiorespiratory interaction we need to have two signals, each corresponding to one of the oscillators (the cardiac and the respiratory oscillators). We construct time series of the (faster) cardiac oscillator from the cutaneous blood flow signal, filtering out all the low frequency content below $0.5 \mathrm{~Hz}$. It is important that FIR filter with linear phase response is used so that the phase information is not distorted. We construct time series of the respiratory oscillator in two different ways: first we take a directly measured respiration signal; and, secondly, we construct the time series by extracting the respiratory related component from the blood flow signal with aid of the of filter.

The choice of a mathematical model for inference is an intricate task. Linear coupling terms were proposed to include the interactions between the cardiac and respiratory oscillatory components at the level of peripheral blood flow. ${ }^{11}$ Here we formulate the model for the cardiac oscillator in the blood flow signal as a Poincaré oscillator driven by the phase of the respiratory oscillator and noise

$$
\begin{aligned}
& \dot{\phi}_{c}=c_{1}^{\phi}+c_{2}^{\phi} \sin \left(\phi_{r}\right)+c_{3}^{\phi} \cos \left(\phi_{r}\right)+\xi^{\phi}(t), \\
& \dot{A}=c_{1}^{A} A+c_{2}^{A} A^{3}+c_{2}^{A} \sin \left(\phi_{r}\right)+c_{3}^{A} \cos \left(\phi_{r}\right)+\xi^{A}(t) .
\end{aligned}
$$

This model cannot describe the whole cardiorespiratory dynamics. We use it as a pilot, trying to describe modulation of the cardiac oscillator by the slow respiratory influence, and trying to infer parameters of this modulation. 

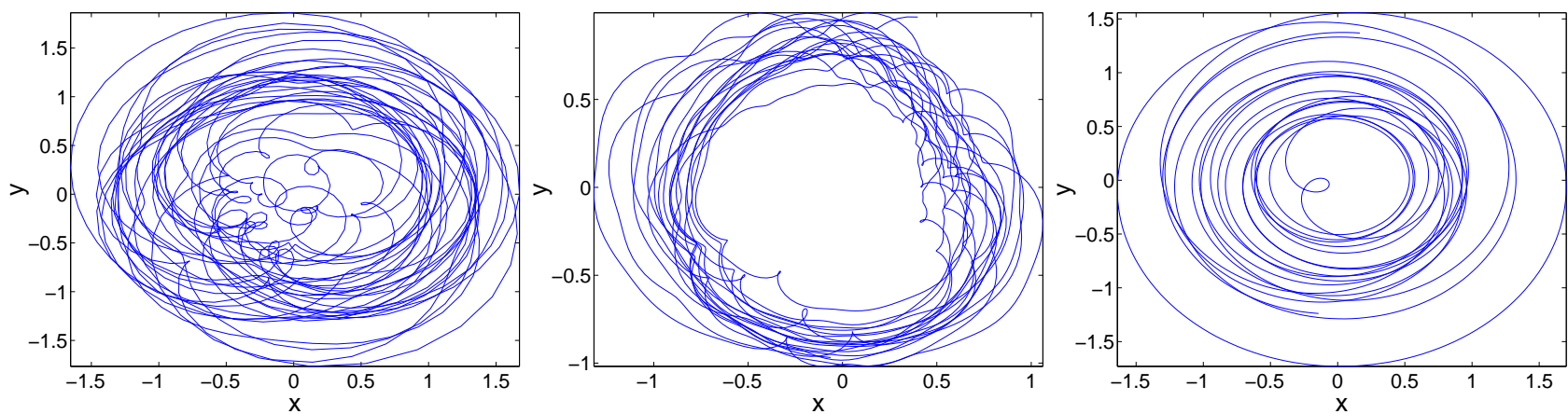

Figure 9. Phase portraits of the cardio (left), original respiratory (middle), and extracted from blood flow signal respiratory (right) oscillators

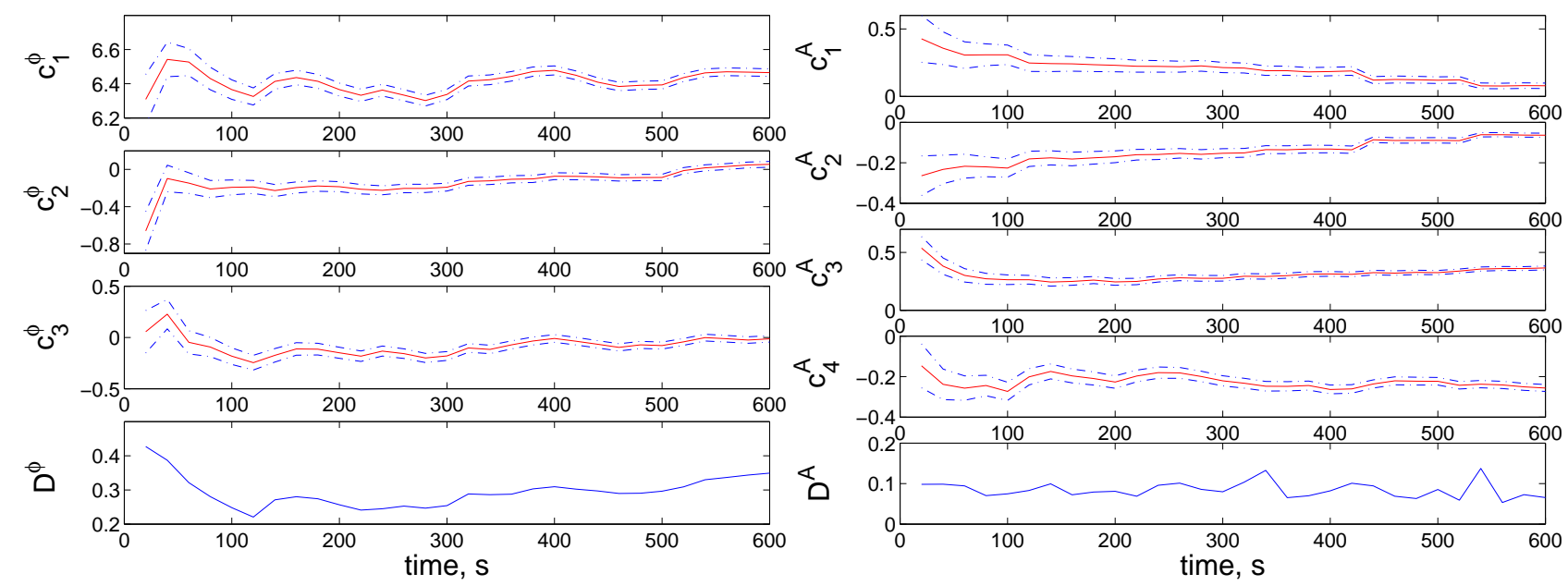

Figure 10. Inference of the cardio oscillator and parameters of the cardiorespiratory modulation due to model (16)-(17) using cardio oscillation extracted from blood flow signal and the original respiration signal

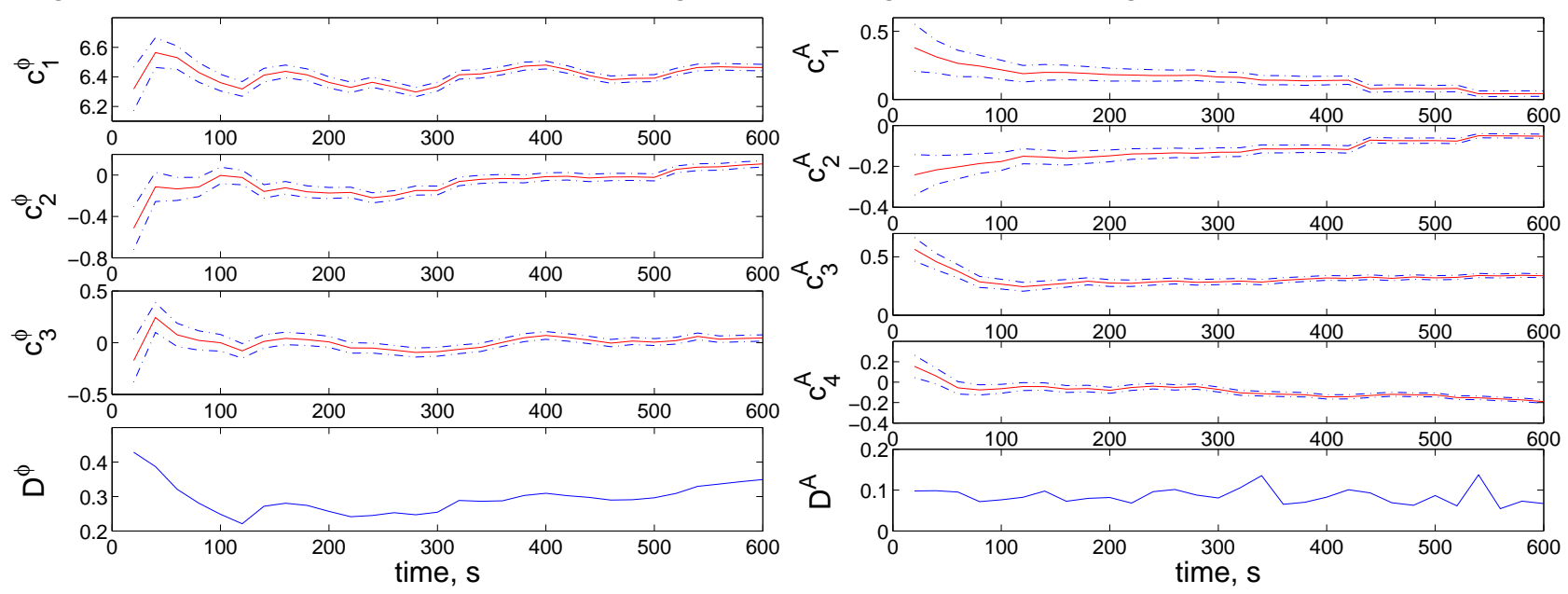

Figure 11. Inference of the cardio oscillator and parameters of the cardiorespiratory modulation due to model (16)-(17) using both cardiac and respiratory oscillations extracted from blood flow signal

The phase portraits of the cardio and the respiratory (constructed in the two different ways) oscillators are shown in Fig. 9. The result of inference is shown in Fig. 10 for the original respiration signal used, and in Fig. 11 for the extracted from blood flow respiration signal. In spite of visual differences in two respiration time series used (compare (middle) and (right) in Fig. 9), the results of inference (Fig. 10 and Fig. 11) are consistent. They 
suggests that, for the signal used, there is significant modulation of amplitude of the cardiac oscillator and there is no noticeable modulation of phase due to respiratory influence.

\section{CONCLUSIONS}

In this paper we have taken advantage of the new Bayesian inference technique, able to deal with stochastic nonlinear systems. We have used it to estimate the parameters of models of the cardiovascular signals directly from the measured time series, as well as demonstrating inference in some model system. There are still many open questions to address. The main and the most difficult problem, which has so far prevented a simple application of the inference scheme to cardiovascular signals, is the absence of any clear understanding of how the cardiovascular signals are generated and how the different physiological mechanisms interact with each other. This means that one of the two necessary parts of the inference technique - the model - is not confidently defined. The choice of model is crucial, and the optimal model means that it should be good enough to capture the basic physiological mechanisms, and that it should be simple enough to have a limited number of unknown parameters, so that they can be reliably inferred.

Much remains to be done in further developing and improving the inference technique. The next important step will be the extension of the inference scheme for systems with non-white noise. There is no reason to suggest that the intrinsic noise in the cardiovascular signals is white, and that is why general properties of noise (not only the noise intensity) have to be inferred from the measured time series. Another important step to be taken will be the introduction of a quantitative measure of success for different models describing the same data set.

However, even at this early stage of the research, it is clear that there is a real possibility of inferring physiologically relevant parameters from the cardiovascular data. Inference offers a very promising tool for the modelling which is likely to contribute significantly to the development of a new diagnostic technique based on noninvasive measurements.

\section{ACKNOWLEDGMENTS}

The work was supported by EPSRC, INTAS, NASA Intelligent Systems/Intelligent Data Understanding program, RFFI, Joy Welch Trust, British Council and the Slovenian Ministry of Education, Science and Sport.

\section{REFERENCES}

1. L. Glass, "Synchronization and rhythmic processes in physiology," Nature 410(6825), pp. 277-284, 2001.

2. A. J. Camm, M. Malik, J. T. Bigger, and et al., "Heart rate variability - Standards of measurement, physiological interpretation, and clinical use," Circulation 93(5), pp. 1043-1065, 1996.

3. A. Stefanovska and M. Bračič, "Physics of the human cardiovascular system," Contemporary Phys. 40(1), pp. 31-55, 1999.

4. S. C. Malpas, "Neural influences on cardiovascular variability: possibilities and pitfalls," Am. J. Physiol.: Heart. Circ. Physiol. 282, pp. H6-H20, 2002.

5. M. Bračič, P. V. E. McClintock, and A. Stefanovska, "Characteristic frequencies of the human blood distribution system," in Stochastic and Chaotic Dynamics in the Lakes, D. S. Broomhead, E. A. Luchinskaya, P. V. E. McClintock, and T. Mullin, eds., pp. 146-153, American Institute of Physics, (Melville, New York), 2000.

6. P. V. E. McClintock and A. Stefanovska, "Noise and determinism in cardiovascular dynamics," Physica A 314, pp. 69-76, 2002.

7. J. V. Ringwood and S. C. Malpas, "Slow oscillations in blood pressure via a nonlinear feedback model," Am. J. Physiol.: Reg. Integr. Compar. Physiol. 280, pp. R1105-R1115, 2001.

8. K. Kotani, K. Takamasu, Y. Ashkenazy, H. Stanley, and Y. Yamamoto, "Model for cardiorespiratory synchronization in humans," Phys. Rev. E 65(5), p. 051923, 2002.

9. M. C. Mackey and L. Glass, "Oscillations and chaos in physiological control systems," Science 197(4300), pp. 287-288, 1977. 
10. P. S. Landa and M. G. Rosenblum, "Modified Mackey-Glass model of respiration control," Phys. Rev. E 52(1), pp. R36-R39, 1995.

11. A. Stefanovska, M. Bračič Lotrič, S. Strle, and H. Haken, "The cardiovascular system as coupled oscillators?," Physiol. Meas. 22(3), pp. 535-550, 2001.

12. A. T. Winfree, The Geometry of Biological Time, Springer-Verlag, New York, 1980.

13. Y. Kuramoto, Chemical Oscillations, Waves, and Turbulence, Springer-Verlag, Berlin, 1984.

14. V. N. Smelyanskiy, D. A. Timucin, D. G. Luchinsky, A. Stefanovska, A. Bandrivskyy, and P. V. E. McClintock, "Time-varying cardiovascular oscillations," in IUTAM Symposium on Nonlinear Stochastic Dynamics, N. S. Namachchivaya and Y. K. Lin, eds., pp. 455-464, Kluwer, Amsterdam, 2003.

15. A. Stefanovska, "Cardiorespiratory interactions," Nonlinear Phenomena in Complex Systems 5(4), pp. 462469, 2002.

16. C. L. Bremer and D. T. Kaplan, "Markov chain Monte Carlo estimation of nonlinear dynamics from time series," Physica D 160(1-2), pp. 116-126, 2001.

17. R. Meyer and N. Christensen, "Bayesian reconstruction of chaotic dynamical systems," Phys. Rev. E 62(3), pp. 3535-3542, 2000.

18. R. Graham, "Path integral formulation of general diffusion processes," Z. Phys. B 26, pp. 281-290, 1977.

19. P. E. McSharry and L. A. Smith, "Better nonlinear models from noisy data: Attractors with maximum likelihood," Phys. Rev. Lett. 83(21), pp. 4285-4288, 1999.

20. M. G. Rosenblum, L. Cimponeriu, A. Bezerianos, A. Patzak, and R. Mrowka, "Identification of coupling direction: Application to cardiorespiratory interaction," Phys. Rev. E. 65(4), p. 041909, 2002. 